\title{
An approach to analyzing the intensity of the daytime surface urban heat island effect at a local scale
}

\author{
Shenlai Xu
}

Received: 30 September 2007 / Accepted: 3 March 2008 / Published online: 14 May 2008

(C) Springer Science + Business Media B.V. 2008

\begin{abstract}
A landscape index LI is proposed to evaluate the intensity of the daytime surface urban heat island (SUHI) effect at a local scale. Three aspects of this landscape index are crucial: the source landscape, the sink landscape, and the contribution of source and sink landscapes to the intensity of the SUHI. Source and sink landscape types are identified using the thermo-band of Landsat 7 with a spatial resolution of $60 \mathrm{~m}$, along with appropriate threshold values for the Normalized Difference Vegetation Index, Modified Normalized Difference Water Index, and Normalized Difference Built-up Index. The landscape index was defined as the ratio of the contributions of the source and sink landscapes to the intensity of the SUHI. The intensity of the daytime SUHI is assessed with the help of the landscape index. Our analysis indicates the landscape index can be used to evaluate and compare the intensity of the daytime SUHI for different areas.
\end{abstract}

S. Xu $(\bowtie)$

State Key Laboratory of Urban and Regional Ecology, Research Center for Eco-Environmental Sciences, Chinese Academy of Sciences,

Beijing 100085, China

e-mail: xushenlai@163.com

S. Xu

Graduate University of Chinese Academy of Sciences,

Beijing 100039, China
Keywords Landscape index - Contribution of source and sink landscapes - Daytime surface urban heat island $\cdot$ Source landscape $\cdot$ Sink landscape

\section{Introduction}

An urban heat island (UHI) is a phenomenon of higher atmospheric and surface temperatures occurring in urban areas compared to the surrounding rural areas. The heat island results from extensive urban surfaces that modify energy and water balances, as well as air movement (Oke 1987). Heat islands are characterized by different layers of the urban atmosphere, and are normally categorized into three groups: canopy layer heat island (CLHI), boundary layer heat island (BLHI), and surface urban heat island (SUHI) groups (Voogt and Oke 2003). The SUHI refers to the relative warmth of urban surfaces compared to surrounding rural areas. It is known the intensity of an urban heat island is stronger at night while that of an SUHI is larger during the daytime (Roth et al. 1989a). The SUHI is typically characterized by the land surface temperature (LST) through the use of satellite thermal infrared remote sensing, which provides a synoptic and uniform means of studying SUHI effects at regional scales. Studies on the SUHI phenomenon using satellite derived LST have been conducted primarily using NOAA and AVHRR data (Gallo and Owen 1998a,b; Streutker 2002, 2003) for regional-scale urban temperature 
mapping. Recently, Landsat Thematic Mapper (TM) and Enhanced Thematic Mapper Plus (ETM+) thermal infrared (TIR) data with 120 and $60 \mathrm{~m}$ spatial resolutions have been used respectively for local-scale studies of UHIs (Chen et al. 2002; Weng 2001). Actually, these studies attempt to investigate and identify the relationship between the landscape type and the SUHI through the application of remote sensing. These methods constitutionally clarified the relationship between the intensity of SUHIs and landscape type. The pattern of LST derived from remotely sensed images is important in the analysis of influence of landscape type on SUHIs. However, this method only considers the influence of the landscape type itself on SUHIs without general evaluation of the intensity of an SUHI on a local scale. Simply comparing the temperature in an urban area to that in the surrounding rural area is not an adequate means of quantifying the intensity of an SUHI at a local or regional scale. Many SUHI analyses treat the quantitative description of landscape type as an end itself and fail to explore the intensity of the SUHI effect at a regional or local scale. A method for analysis at these scales is needed. The purpose of this paper is to outline a new landscape index to evaluate the intensity of the SUHI effect at a local scale. The landscape index will be a perfect combination of the SUHI effect and landscape pattern, and is aimed at better understanding the mechanism of the SUHI effect. It will stimulate discussion, refinements, replacements, additions, hypotheses, research and further understanding.

\section{Data and method}

Study area and data

Beijing is located between $115^{\circ} 50^{\prime}-116^{\circ} 59^{\prime} \mathrm{E}$ and $39^{\circ}$ $36^{\prime}-40^{\circ} 23^{\prime} \mathrm{N}$ (Fig. 1), approximately covering $16,800 \mathrm{~km}^{2}$. This area covers nine counties and ten districts. Previous study on the urban environment

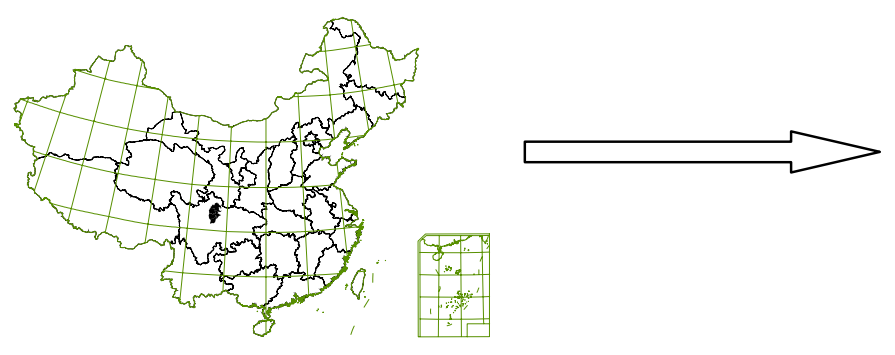

Chinese borders

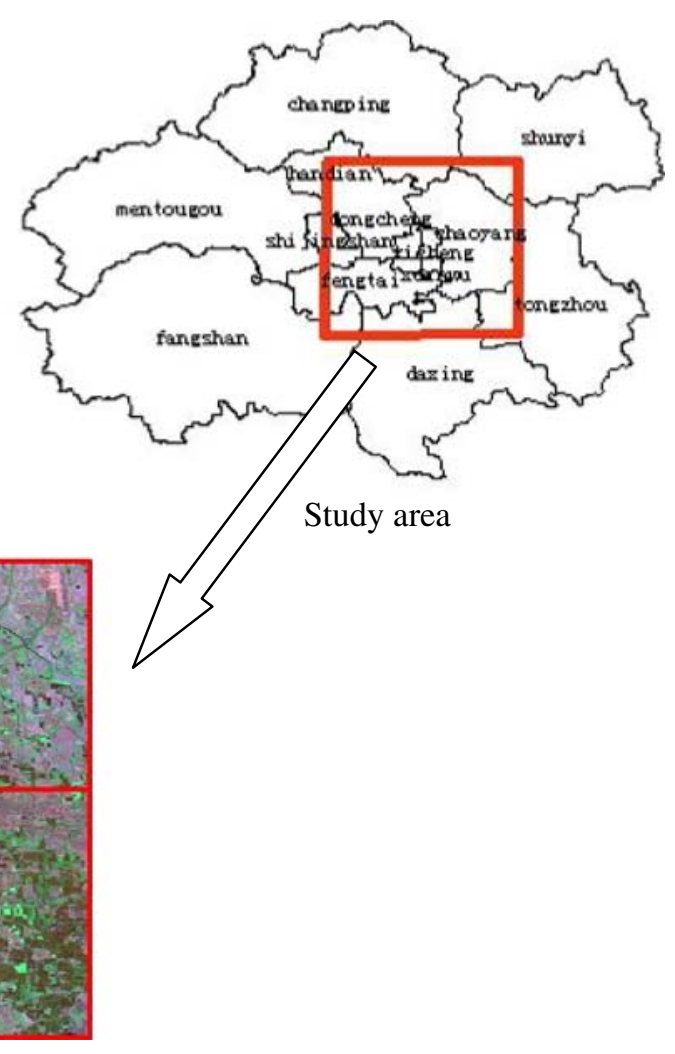

Image of the study area

Fig. 1 Location of the study area 
using satellite or ground data has shown the UHI in Beijing intensified from 1996 to 2000 . The average UHI intensity reached $3.3^{\circ} \mathrm{C}$ (Wang et al. 2006). In recent decades, a diversified and growing economy has continuously attracted new residents and stimulated urban growth, especially in the main developed districts (red rectangle in Fig. 1). These regions are selected as the study area, and comprise $16 \mathrm{~km}^{2}$ of flat landform. The diversification of land use types combined with growing urbanization makes it an ideal area to analyze SUHI effects.

Method

\section{Image pre-processing}

Many SUHI studies have been conducted using thermal data from satellites (Aniello et al. 1995; Streutker 2002; Lo and Quattrochi 2003; Dousset and Gourmelon 2003). These studies, employing medium scale sensors (Landsat TM) are more feasible than studies using data from meteorological station networks, and can provide a spatial view of the SUHI over large urban areas.

To quantitatively measure the LST and compare SUHI intensity in the study area, Landsat 7 ETM+ images (Row032/Path123), acquired on July 1, 1999, were analyzed. SUHIs are usually studied using satellite data of high spatial resolution acquired during daytime when heat island intensities are greatest (Roth et al. 1989b). The average maximum SUHI of Beijing is strong in summer and weak in autumn and winter (Wang and Wang 2006). Therefore the acquired data is ideal for analyzing the SUHI. Image bands $1-5$ and 7 have a spatial resolution of $30 \mathrm{~m}$, whereas band 6 consists of a $60-\mathrm{m}$ resolution coarse grain thermal band $(8-12 \mathrm{~mm})$. An approximate surface temperature can be extracted from the latter. Temperature data was extracted from the coarse grain band as it had a less striped texture and a wider range of recorded digital number (DN) values. The technique developed by the Landsat science team was chosen for the conversion. Calculations for each pixel were made using the image processing software ENVI and thermal image "p123r032_7k19990701_z50_ban62," which is the coarse grain band 6 of the Landsat 7 ETM+ data. This TIR band was rectified to the Universal Transverse Mercator projection system (ellipsoid GRS80, datum D_WGS_1984, zone 50) and georeferenced to a relief map using approximately 60 ground control points, primarily river intersections, evenly distributed across the image. A first order polynomial model was used for the rectification with nearest neighbor resampling. The root mean square (RMS) errors were less than 0.25 pixels $(7.5 \mathrm{~m})$ for each of the four images. The original DN of the ETM+ images were converted to ex-atmospheric reflectance based on the methods provided by Chander and Markham (2003).

Noise reduction is necessary for remotely sensed images, especially for the TIR band. The noise may affect the retrieval of LST. There is periodic noise (e.g., stripes in the $\mathrm{TM} /$ band 6) and non-periodic noise (e.g., speckles). In this study, a self-adaptive filter method was used to remove non-periodic noise and a fast Fourier transformation was used to automatically remove periodic noise. The removal of both periodic and non-periodic noise was performed using ERDAS Imagine 8.6 software.

\section{Retrieval of LST from the Landsat 7 ETM+ images}

Satellite TIR sensors measure radiances at the top of the atmosphere, from which brightness temperatures (also known as blackbody temperatures) can be derived using Plank's law (Dash et al. 2002). It is recognized that the water vapor content does vary over time due to seasonality and inter-annual variability of the atmospheric conditions. It is not appropriate to directly compare at-satellite brightness temperatures for multiple temporal periods. Hence, the image for a single time period acquired on July 1, 1999, was selected to avoid the influence of the atmosphere on radiance temperature. Accurate determination of the surface temperature also requires correction for atmospheric interference. However, the error in surface temperature due to an error in the emissivity correction is twice that due to an error in the atmospheric correction (Prata et al. 1995). Considering this study is interested in relative surface temperature differences between source landscapes and sink landscapes, the error due to the atmospheric effects is not corrected. Since the SUHI effect is studied to a spatial extent of a few kilometers $\left(16 \mathrm{~km}^{2}\right)$ around the city center (local scale), it is reasonable to assume homogeneity of the atmosphere over the urban area. The error produced in the LST subjected to the influence of atmospheric conditions is uniform across the image and the influence of the 
atmosphere on radiance temperature can be neglected. Therefore the at-satellite brightness temperature can be used to reflect the distribution of the surface temperature fields.

The ETM+ TIR band $(10.4-12.5 \mu \mathrm{m})$ data were used to derive the LST. The spatial distribution of the daytime LST in the study area was identified (Fig. 4a).

A thermal band image data calibration is performed in two-steps (Chander and Markham 2003):

(a) conversion of the $\mathrm{DN}$ values of band 6 into spectral radiance $L\left(\mathrm{~W} /\left(\mathrm{m}^{2} \mathrm{sr} \mu \mathrm{m}\right)\right)$ using

$L=\frac{L_{\text {max }}-L_{\text {min }}}{Q_{\text {cal max }}-Q_{\text {cal min }}} \times\left(Q_{\text {cal }}-Q_{\text {calmin }}\right)+L_{\text {min }}$

and (b) conversion of the spectral radiance $L$ to atsensor brightness temperature BT in Kelvin. The conversion formula is given by

$\mathrm{BT}=\frac{K_{2}}{\left[\ln \left(\frac{K_{1}}{L}+1\right)\right]}$

where BT is the at-sensor brightness temperature $(\mathrm{K})$, $K_{2}$ is a calibration constant $(1,282.71 \mathrm{~K}), K_{1}$ is another calibration constant $\left(666.09 \mathrm{~W} \mathrm{~m}^{-2} \mathrm{sr}^{-1}\right.$ $\mathrm{lm}^{-1}$ ) and $L$ is the spectral radiance at-sensor (W $\left.\mathrm{m}^{-2} \mathrm{sr}^{-1} 1 \mathrm{~m}^{-1}\right)$.

Brightness temperature derived from Eq. 2 is the temperature that a blackbody would obtain in order to produce the same radiance at the same wavelength $(k=$ $11.5 \mathrm{~lm})$. Therefore, additional correction for spectral emissivity is required to account for the non-uniform emissivity of the land surface. The emissivity correction was carried out using surface emissivities for the specified land covers derived from the methodology described in Stathopoulou et al. (2007). The procedure involved combining surface emissivity maps obtained from the normalized difference vegetation index (NDVI) thresholds method (Sobrino and Raissouni 2000). Thus, the emissivity corrected LST Ts is calculated as

$\mathrm{TS}=\frac{K 2}{\left[1+\ln \left(\frac{\lambda \cdot \mathrm{BT}}{\rho} \cdot \ln \varepsilon\right)\right]}$

where TS is the land surface temperature $(\mathrm{K}), \lambda$ is the wavelength of emitted radiance $(11.5 \mathrm{~lm}) ; \rho$ is $h \times c / r=1.438 \times 10^{-2} \mathrm{mK}$ and $\varepsilon$ is the spectral surface emissivity.
Derivation of NDVI, NDBI, and MNDWI from ETM+ imagery

The NDVI (Gao 1996), modified normalized difference water index (MNDWI; Xu 2005), and normalized difference built-up index (NDBI; Zha et al. 2003) were used to characterize the land use/cover types in the study area. The NDVI of Eq. 4 was generally used to express the density of vegetation (Purevdorj et al. 1998).

$\mathrm{NDVI}=(\rho($ band 4$)-\rho($ band 3$)) /(\rho($ band 4$)+\rho($ band 3$))$

The NDBI of Eq. 5 was introduced in this study and is sensitive to the built-up area.

$\mathrm{NDBI}=(\rho($ band5 $)-\rho($ band 4$)) /(\rho($ band 4$)+\rho($ band5 $))$

The MNDWI can significantly enhance the water information, especially in the area with mostly builtup land as background. It can dim the built-up land information effectively while highlighting water information, and accurately extract the water body from the study areas (Fig. 2).

$\operatorname{MNDWI}=(\rho($ band 2$)-\rho($ band5 $)) /(\rho($ band 2$)+\rho($ band5 $))$

A new landscape index

The basic framework in this landscape index is as follows.

1. During pattern-processing, different landscape types can be divided into two types of landscape: source landscapes and sink landscapes. A source landscape is a landscape that contributes positively to the development of the ecological process, whereas a sink landscape is one that is unhelpful.

2. Source and sink areas embracing dual information on landscape pattern and the ecological process independent of scale are a new description for the intensity of the SUHI at a local scale.

3. Vegetation, greenery, and water are defined as sink landscapes due to their negative contribution to the 
Fig. 2 Methodology

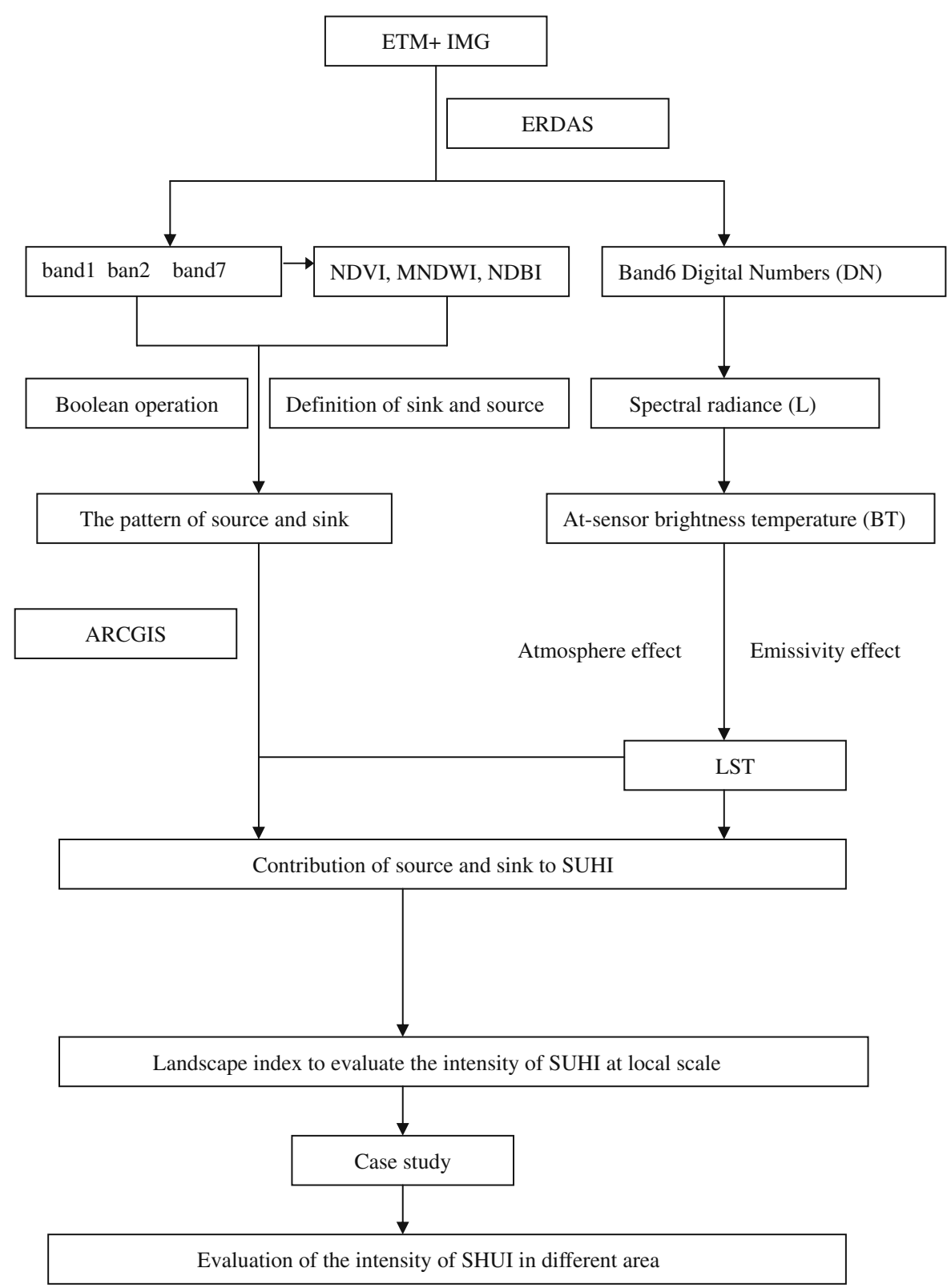

SUHI, while built-up areas are defined as source landscapes due to their positive contribution.

4. The contribution of source and sink landscapes to the SUHI determines the intensity of the SUHI at a local scale.

5. The evaluation of the intensity of the SUHI at a local scale is executed under the condition that climatic factors such as atmospheric temperature, relative humidity, wind direction and wind speed are assumed constant.

\section{SUHI characteristics of source and sink landscapes}

Source and sink landscapes are defined as having specified processes. The terms "source" and "sink" are introduced into research on the configurationprocess relationship as landscape units. A source landscape acts as a sponsor of a specified process and a sink landscape acts as a terminator. A source landscape refers to a landscape unit promoting the ecological process and a sink landscape refers to a 
landscape unit weakening the process. Source and sink landscapes need to be defined with regard to a specific ecological process (Chen et al. 2006a), since a landscape type could be a source area for one ecological process and a sink area for another. The contribution of a source landscape is completely positive and the contribution of a sink landscape is completely negative for a specific process.

Vegetation, greenery, and water bodies may be considered as sink landscapes for the SUHI due to their weakening effect (Chen et al. 2006b); industrial areas, commercial areas, airports, and residential areas are source landscapes because of their positive effect (Chen et al. 2006a). In this study area, built-up areas were defined as source landscapes while vegetation, greenery and water were defined as sink landscapes.

Indices of NDVI, MNDWI, and NDBI are used in the classification of source and sink areas. However, it was difficult to determine the appropriate threshold values for the indices to obtain accurate classification. For this purpose, the relative spectral characteristics of source and sink landscapes were analyzed, and the Landsat 7 ETM+ image on July 7, 1999, was taken as an example for the spectral analysis. Through repeated sampling of the representative pixels of sources and sinks, the values in all seven bands were obtained

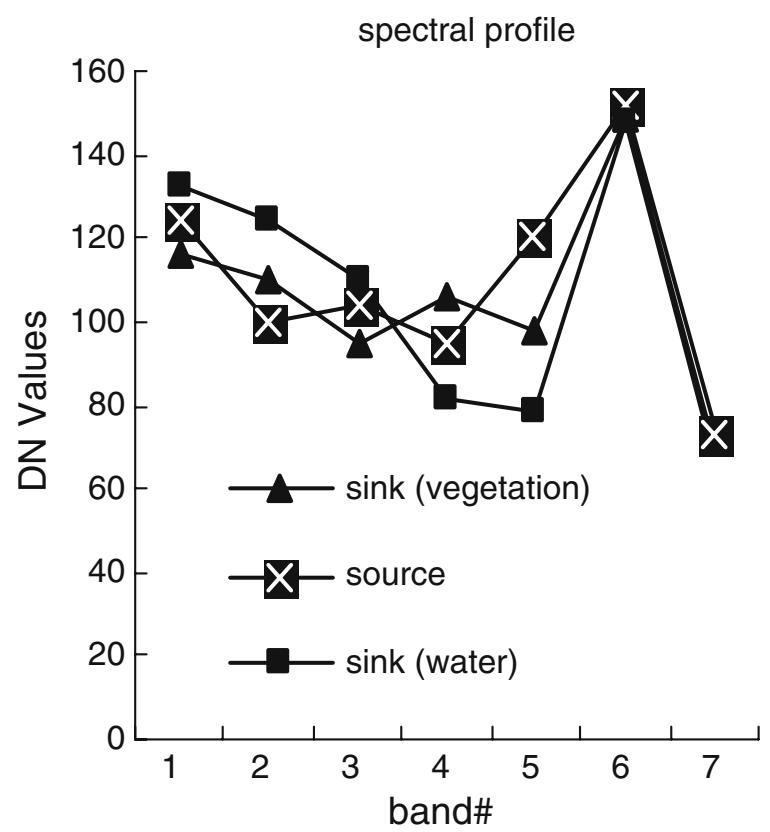

Fig. 3 Spectral profiles of source and sink landscapes in the study area
Table 1 Indices, values, and ranges for source and sink

\begin{tabular}{llll}
\hline Landscape type & NDWI & NDBI & MNDWI \\
\hline Sink landscape (vegetation) & $>0$ & $<0$ & $>0$ \\
Sink landscape (water) & $<0$ & $<0$ & $>0$ \\
Source landscape & $<0$ & $>0$ & $<0$ \\
\hline
\end{tabular}

and averaged (Fig. 3). After the spectral characteristics of source and sink landscapes were identified, Boolean operators were used on the spectral bands to improve the results of the indices (Chen et al. 2006b). An operator (band 4-band 5) $>0$ was combined with (band 4-band 3) $>0$ (or NDVI $>0$ ) to extract vegetation (sink areas) from the Landsat imagery because only vegetation has these spectral features. Similarly, water (sink areas) was extracted by combining (band 3-band 4) $>$ and (band 2-band 5) $>0$ with the MNDWI. Sources were derived by combining (band 4-band 3) $<0$ and (band 4 -band 5) $<0$ with the NDBI (Table 1). The threshold approach in combination with the Boolean operators based on band DN values and indices worked well in identifying source and sink types. Compared with traditional image classification methods, such as automated supervised and unsupervised classifications, our method achieved relatively high accuracy. The sampling points were randomly taken across the study area, and were relatively uniformly distributed among sources and sinks. The overall accuracy of the classification was approximately $90 \%$.

\section{Contribution of sources and sinks to the intensity of the SUHI}

The source landscapes cover built-up areas such as industrial areas, commercial areas, the airport, and residential areas and the sink landscapes cover vegetation, greenery, and water. In this study, the contribution of sink and source landscapes to the daytime SUHI is calculated according to the acquisition time of the image. Considering the need to construct the landscape index, we incorporated builtup areas into source landscapes, and merged vegetation, greenery, and water into a single sink landscape type. The mean temperatures of source and sink landscapes were calculated by averaging for all pixels of a given source or sink landscape. We can use the difference in temperature between source landscape 
$319 \mathrm{~K}$
$294 \mathrm{~K}$
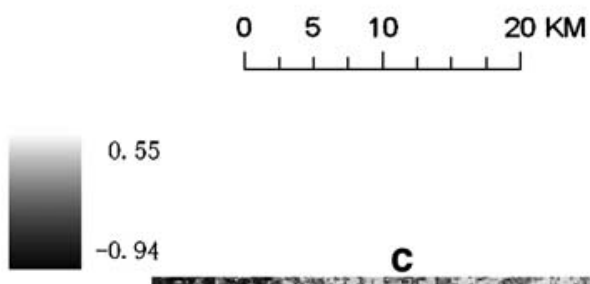

$20 \mathrm{KM}$

0.55
-0.94
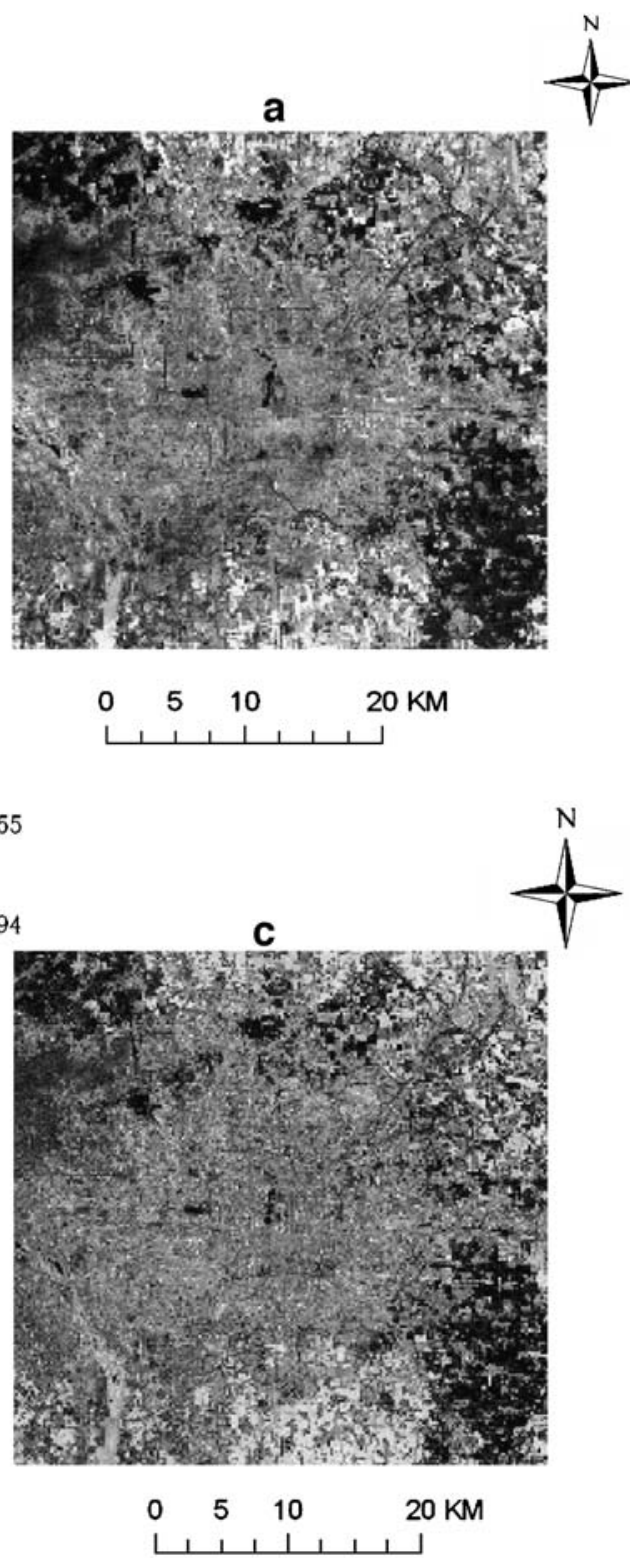

0.54

$-0.44$

b

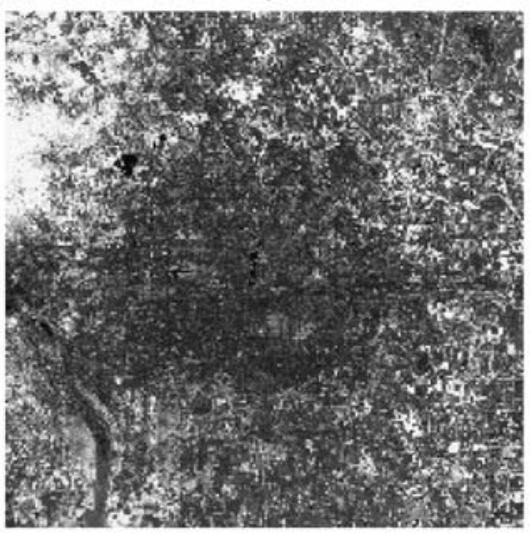

1

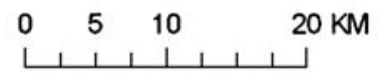

0.97
-0.47
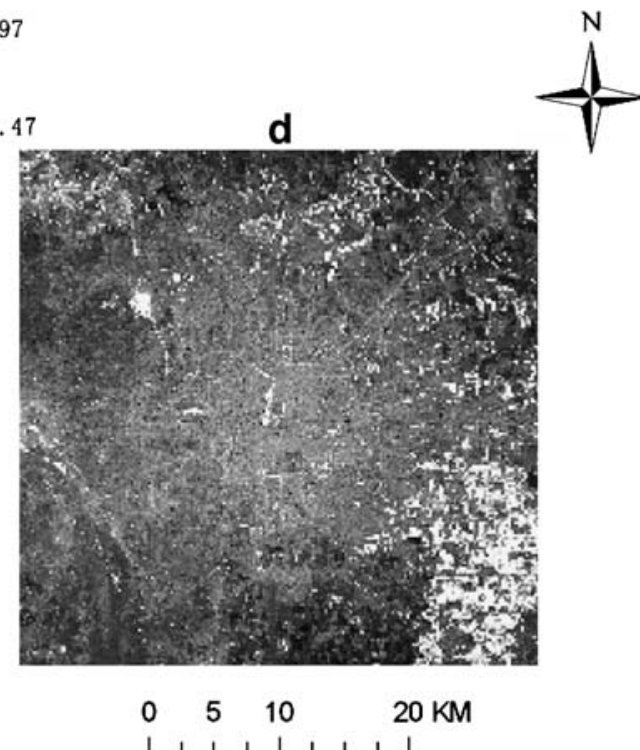

Fig. 4 a LST, b NDVI, c NDBI, and d MNDWI, derived from ETM+ in the study area

and the whole area to define its positive contribution to the SUHI. The difference in temperature between sink landscape and the whole area was also calculated to determine its negative contribution to the SUHI. The contribution of the source landscape is the fraction of the entire region that is source landscape multiplied by the difference in temperature between the sink landscape and the entire region (Chen et al. 2006a). Similarly, the contribution of the sink landscape is the fraction of the entire region that is sink landscape multiplied and the difference in temperature between sink landscape and the entire region (Chen et al. 2006b). Thus the contribution index is proposed as

Contribution Index $=D_{t} \times S$

where $D_{t}$ represents the difference in the temperature between the sink or source landscape and the entire region; $S$ represents the fraction of the area that is 
Table 2 Characteristics of the source and sink in the study area

\begin{tabular}{lllll}
\hline Type*** & $\mathrm{S}(\%)$ & Temp(K) & $T_{\text {source }}-T_{\text {sink }}(\mathrm{K})$ & $\begin{array}{l}\text { Regional } \\
\text { temp(K) }\end{array}$ \\
\hline Sink & 50 & 303.96 & 1.361 & 304.64 \\
Source & 50 & 305.32 & & \\
\hline
\end{tabular}

Type*** refers to the compatible landscape type

source landscape or sink landscape in the entire area. For a source landscape, $D_{t}$ is positive, and thus the contribution index is also positive, whereas for a sink landscape, the values are negative.

\section{Evaluation of the intensity of the SUHI}

The intensity of the SUHI at a local scale is determined by the contribution of the source or sink landscape. To determine the intensity of the SUHI, the landscape index LI is defined as the absolute value of the ratio of the contribution index of the sink and source $\left(\left|\mathrm{CI}_{\text {sink }} / \mathrm{CI}_{\text {source }}\right|\right)$. With the help of the landscape index, we can determine and compare the intensity of the SUHI in different areas at a local scale. The formula is given by

$\mathrm{LI}=\left|\mathrm{CI}_{\text {sink }} / \mathrm{CI}_{\text {source }}\right|$

where CI represents the contribution of the source or sink landscape to the intensity of the SUHI. When the value of LI is higher than 1, the contribution of the source and sink landscapes weakens the intensity of the SUHI. When the value of LI is less than 1, the contributions of the source and sink landscape

Table 3 Characteristics of the source and sink in areas A, B, C, and D

\begin{tabular}{llllll}
\hline Id & Type*** & $S(\%)$ & Temp (K) & $T_{\text {source }}-T_{\text {sink }}(\mathrm{K})$ & $\begin{array}{l}\text { Regional } \\
\text { temp (K) }\end{array}$ \\
\hline A & Sink & 52 & 302.47 & 2.748 & 303.80 \\
& Source & 48 & 305.24 & & \\
B & Sink & 52 & 304.10 & 0.131 & 305.16 \\
& Source & 48 & 306.14 & & \\
C & Sink & 40 & 303.76 & 1.75 & 304.45 \\
& Source & 60 & 305.51 & & 305.09 \\
D & Sink & 62 & 304.73 & 0.597 & \\
& Source & 38 & 305.32 & & \\
\hline
\end{tabular}

Type $^{* * *}$ refers to the compatible landscape type
Table 4 Parameters of the landscape index in samples A, B, C, and D

\begin{tabular}{llllrl}
\hline Id & Type*** & $S(\%)$ & $D_{t}(\mathrm{~K})$ & \multicolumn{1}{c}{ CI } & LI \\
\hline A & Sink & 52 & 1.33 & -0.69 & 1 \\
& Source & 48 & 1.44 & 0.69 & \\
B & Sink & 52 & 1.06 & -0.55 & 1.17 \\
& Source & 48 & 0.98 & 0.47 & \\
C & Sink & 40 & 0.69 & -0.28 & 0.44 \\
& Source & 60 & 1.06 & 0.64 & \\
D & Sink & 62 & 0.37 & -0.23 & 2.56 \\
& Source & 38 & 0.23 & 0.09 & \\
\hline
\end{tabular}

Type $^{* * *}$ refers to the compatible landscape type

promote the intensity of the SUHI. When the value of LI is 1 , the intensity of the SUHI is unchanged. Different values of LI represent different degrees of weakening or promoting the intensity of SUHI at a local scale.

\section{Case study of downtown Beijing}

The method is applied to the central $16 \mathrm{~km}^{2}$ of the Beijing metropolitan area. The area was divided into four $4 \mathrm{~km}^{2}$ areas (areas A, B, C, and D; Fig. 1).

The pattern of the source/sink landscape Figs. 5a and 7 was derived from a Landsat 7 ETM+ image

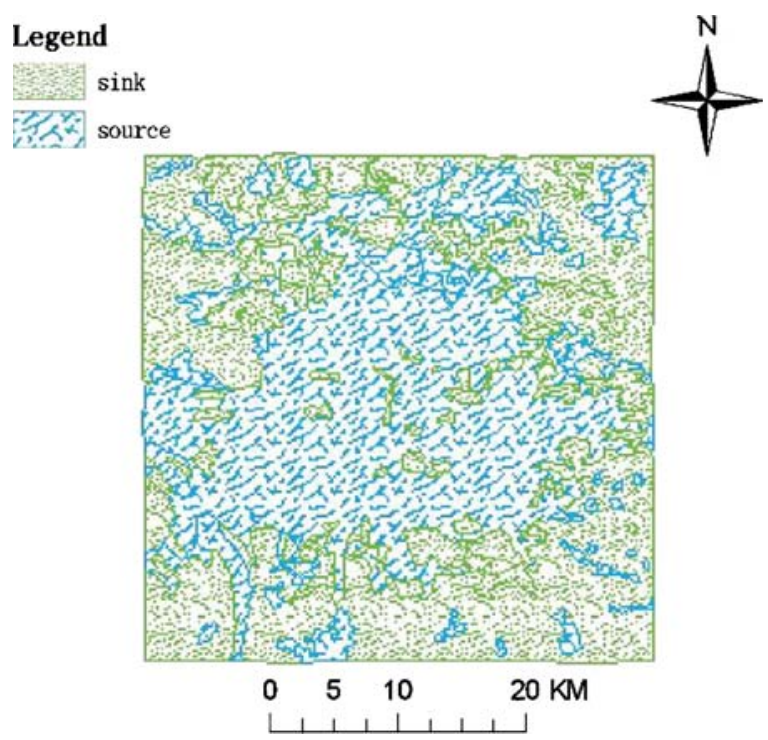

Fig. 5 Distributions of sink and source landscapes 
taken in July with appropriate threshold values for the indices of NDVI, NDBI, and MNDWI. Figs. 4a and 6 illustrate the spatial distribution of the LST for the selected areas; dark to bright tones indicate cooler to warmer LSTs. Mean surface temperatures for the source and sink landscapes are given in Tables 2 and 3. The values of $\mathrm{CI}$ for source and sink landscapes in the study areas A, B, C, and D were calculated and are presented in Table 4.
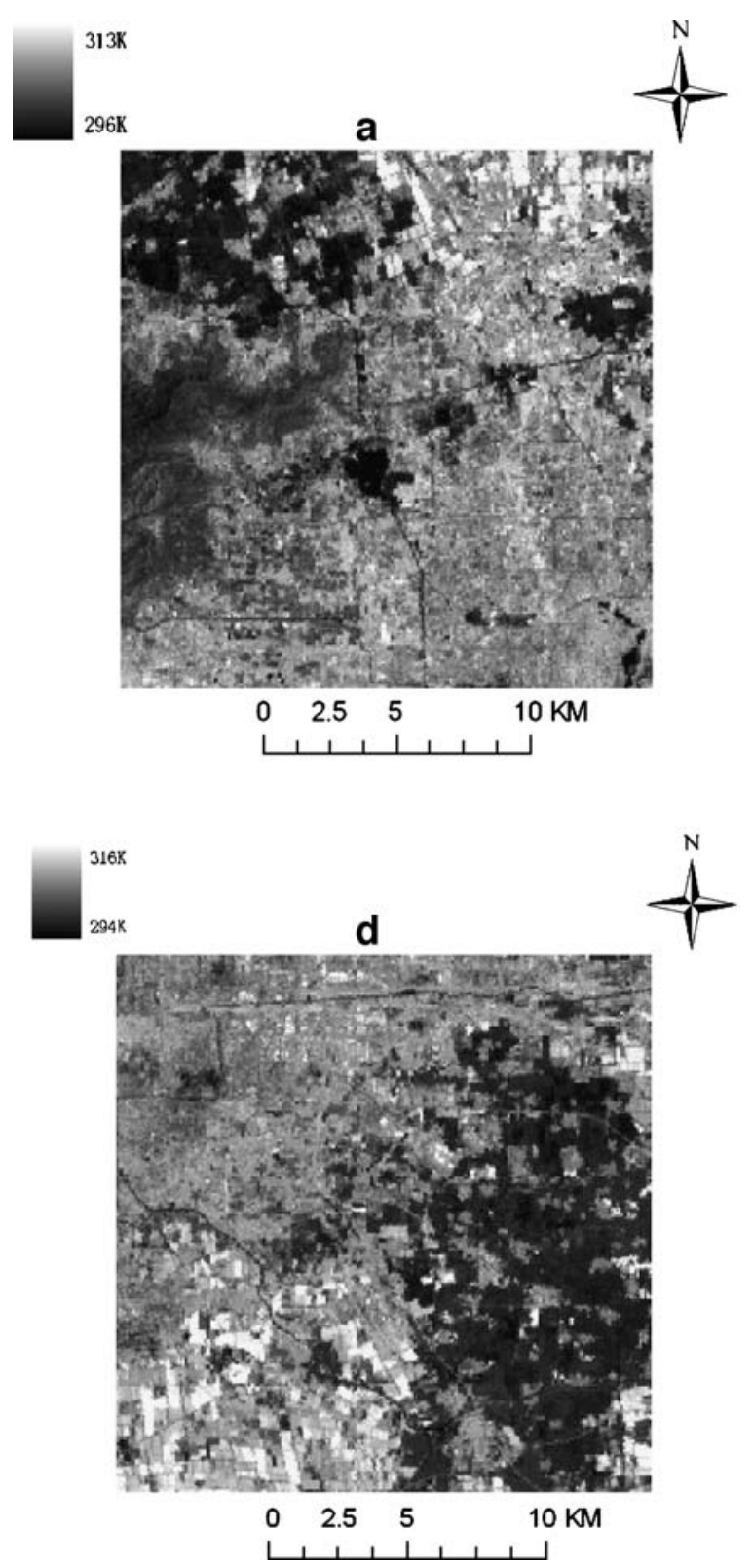

\section{Results and discussion}

Characteristics of sources and sinks in the sample areas

The digital remote sensing method provides not only the spatial distribution of the source and sink landscapes in the study area of $16 \mathrm{~km}^{2}$ (Fig. 5a), but also the spatial distribution of LST for the source and sink
$314 \mathrm{~K}$
$297 \mathrm{~K}$ $214 \mathrm{~K}$
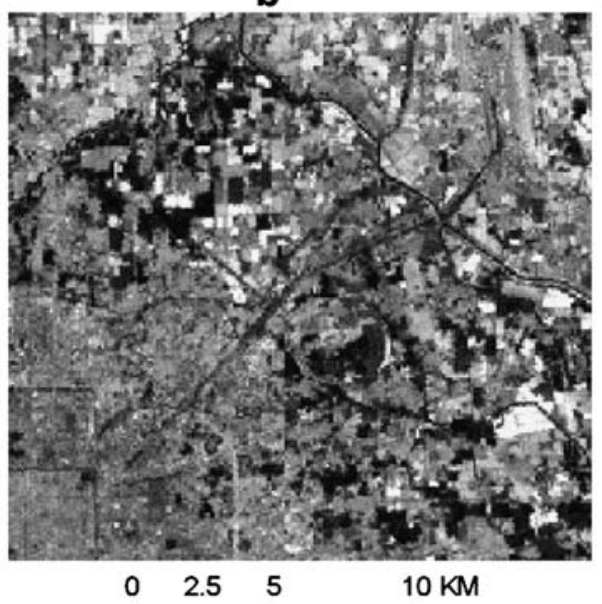

$10 \mathrm{KM}$
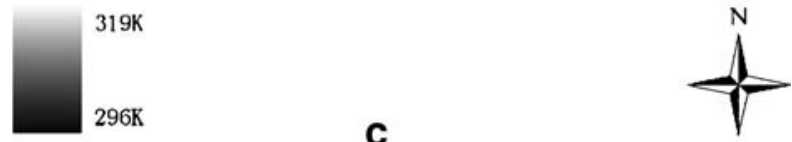

Fig. 6 Distribution of LST in sample areas A (a), B (b), C (c), and D (d) derived from ETM+ 
areas (Fig. 4a). The daytime LST map of the $16 \mathrm{~km}^{2}$ study area shows a clear SUHI effect by contrasting surface temperatures (Fig. 4a). The daytime LST map had a range of 294-319 K with higher surface temperatures for the source landscape and lower temperatures for the sink landscape. Mean temperatures for the source and sink landscapes were calculated by averaging all pixels of a given source or sink landscape. The daytime LST for the source landscape was $1.361 \mathrm{~K}$ higher than that of the sink landscape, and the mean temperature of the $16 \mathrm{~km}^{2}$ was $304.64 \mathrm{~K}$, as shown in Table 2.

The LI for the four $4 \mathrm{~km}^{2}$ areas with different landscape configurations were calculated to evaluate the intensity of the SUHI in the sample areas. The summer LST map for areas A, B, C and D had ranges of 296-313, 297-314, 296-319, and 294-316 K, respectively, with higher surface temperatures located in the source landscape and lower temperatures located in the sink landscape. The average tempera-
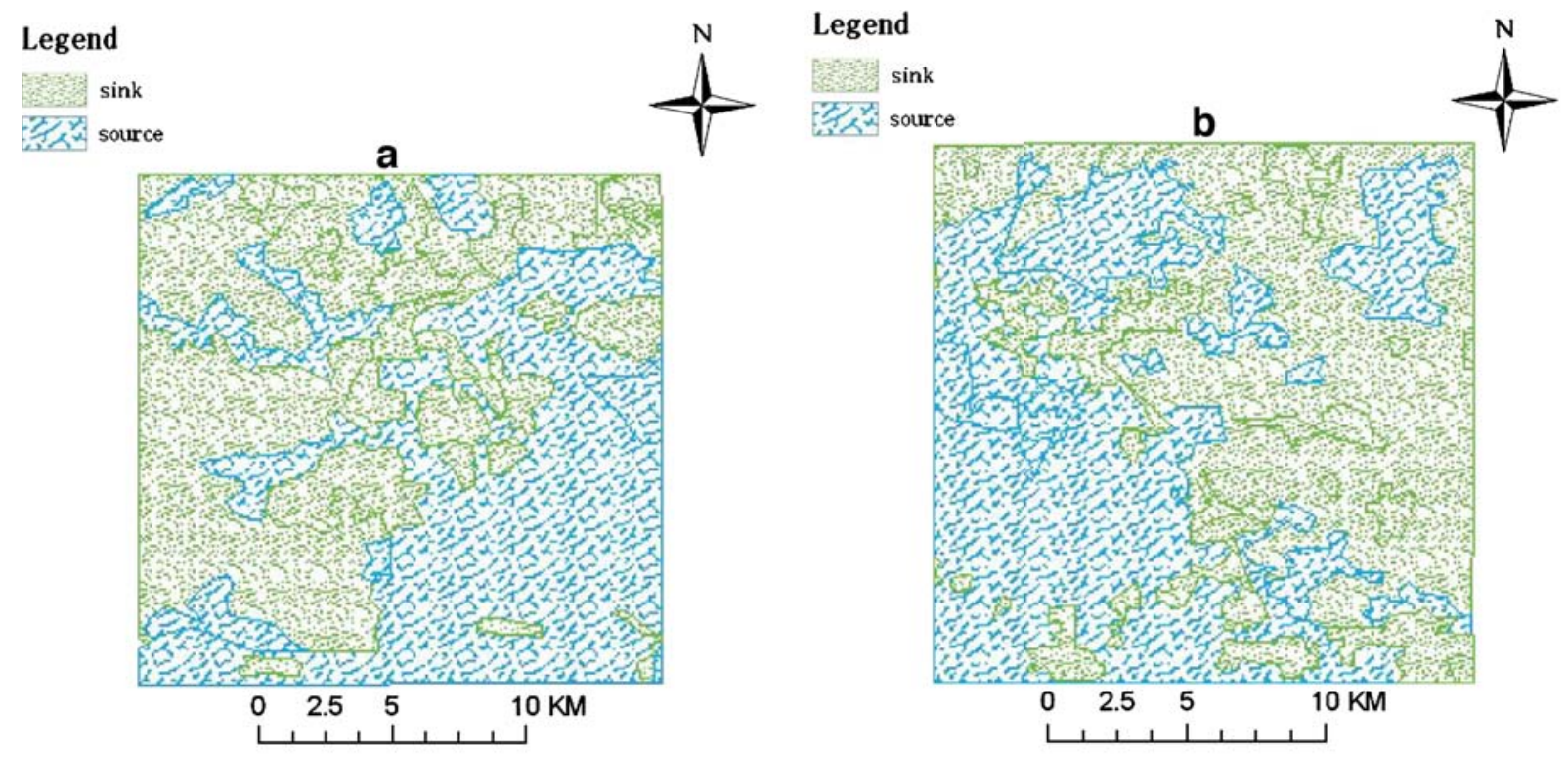

\section{Legend}

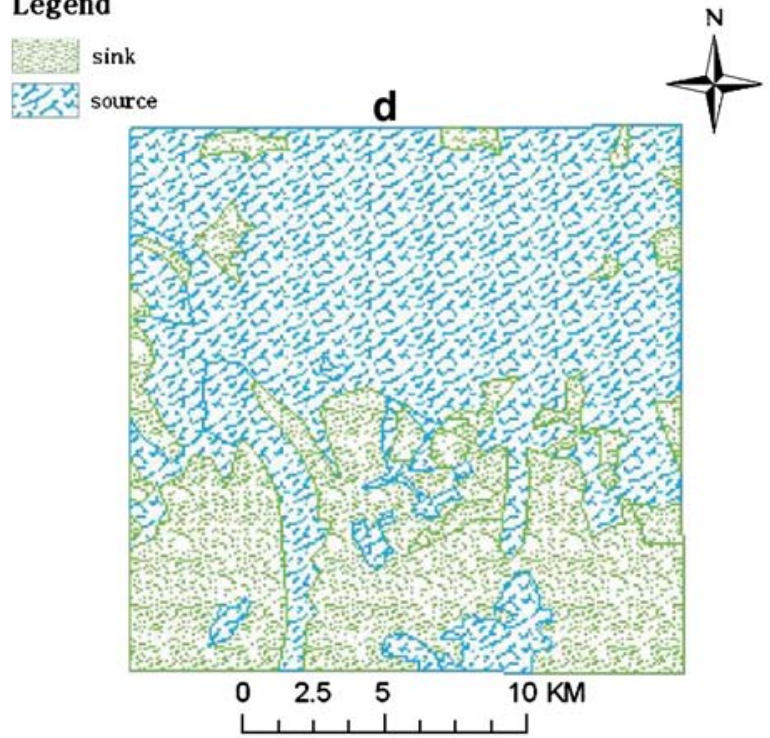

\section{Legend}

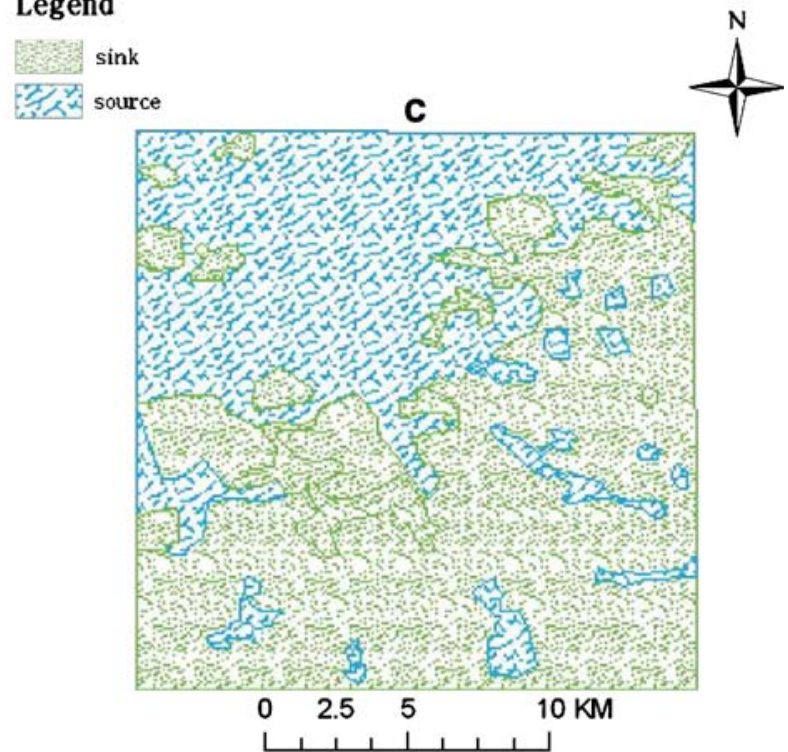

Fig. 7 Distributions of source and sink landscapes in sample areas A (a), B (b), C (c), and D (d) 
ture for the source and sink landscapes is found by combining the surface temperature data (in raster format; Fig. 6) with the reproduced source/sink data (in vector format; Fig. 7). In particular, the source and sink map (polygon layer) and the surface temperature image of the city are superimposed and mean surface temperatures are computed for the source and sink landscapes using a geographical information system polygon operation.

For area A, there was a mean temperature of $301.60 \mathrm{~K}$. The quantitative analysis results reflect that the source landscape has a higher daytime mean surface temperature $(305.24 \mathrm{~K})$ than the sink landscape (302.49 K).

For area B, there was a mean temperature of $305.10 \mathrm{~K}$ and the source landscape had a higher daytime mean surface temperature $(306.14 \mathrm{~K})$ than the sink landscape (304.10 K).

For area $\mathrm{C}$, there was a mean temperature of $304.45 \mathrm{~K}$ and the source landscape had a much higher daytime mean surface temperature $(305.51 \mathrm{~K})$ than the sink landscape (303.76 K).

For area $\mathrm{D}$, there was a mean temperature of $303.40 \mathrm{~K}$ and the source landscape had a much higher daytime mean surface temperature $(305.32 \mathrm{~K})$ than the sink landscape (304.76 K).

The daytime MST of the source landscape in sample areas A, B, C, and D tended to be higher than that of the sink landscape but to different extents. A source landscape has the characteristic of being a large exposed concrete surface area that increases the temperature because of high heat absorption. A sink landscape is a large area of evaporating surfaces. The landscape puts more energy into sensible heat and less into latent heat. Specifically, vegetation and water permeable surfaces reduce the surface temperature through evapotranspiration.

Contributions of source and sink landscapes

to the SUHI in the study area

We can use the difference in temperature between the source and sink landscapes and the entire region to measure the increase or decrease in SUHI strength. $D_{t}$ values for the sink and source landscapes in areas $\mathrm{A}$, $\mathrm{B}, \mathrm{C}$, and D were 1.33 and $1.44,1.06$ and $0.98,0.69$ and 1.06 , and 0.37 and 0.23 , respectively. $S$ values for sink and source landscapes were 52\% and 48\%, 52\% and $48 \%, 40 \%$ and $60 \%$, and $62 \%$ and $38 \%$, respectively (Table 4), considering the difference in the percentage areas of the source and sink landscapes. For the source landscape, the contribution to the intensity of the SUHI is the fraction of the area that is part of the source landscape multiplied by the difference in temperature between the sink landscape and the entire region. Similarly, for the sink landscape, the contribution to the intensity of the SUHI is the fraction of the area that is part of the sink landscape multiplied by the difference in temperature between the sink landscape and the entire region. For areas $\mathrm{A}, \mathrm{B}, \mathrm{C}$, and $\mathrm{D}$, the values of $\mathrm{CI}$ for the source landscape were $0.69,0.47,0.64$, and 0.09 . The values of CI for the sink landscape were $-0.69,-0.55,-0.28$, and -0.23 (Table 4 ).

Evaluation of the intensity the SUHI in the study area

The values of LI in areas A, B, C, and D were calculated and are presented in Table 4. The value of LI was higher than 1 in areas B and D, which implies the combined contribution of the source and sink landscapes weakens the SUHI. Likewise, the value of $\mathrm{W}$ was lower than 1 in area $\mathrm{C}$, which indicates the contribution of the source and sink landscapes promotes the surface urban heat. From Fig. 8, different values of LI in areas A, B, C, and D imply different magnitudes of the intensity of the SUHI in these sample areas. The most intensified SUHI was 2.56 in area D, and the least intensified SUHI was 0.44 in area $\mathrm{C}$.

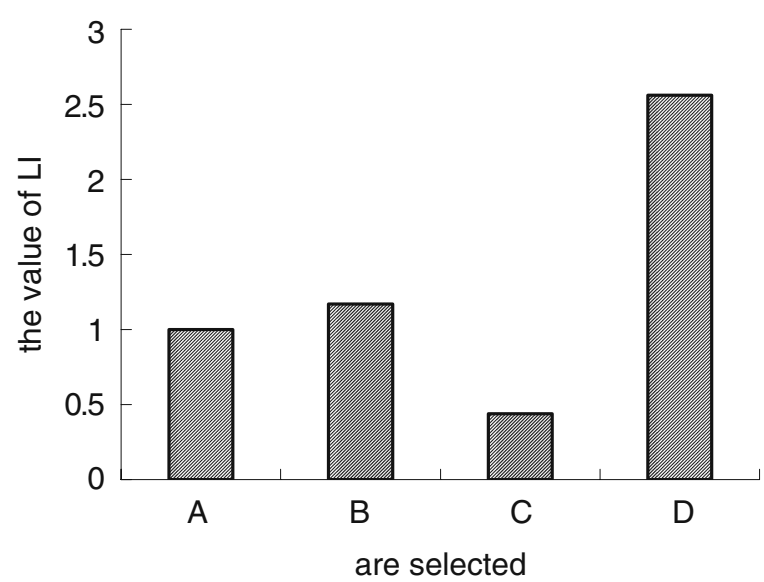

Fig. 8 Values of the landscape index (LI) in the area selected 


\section{Conclusion}

In this study, a landscape index for estimating the daytime SUHI intensity of urban areas was presented. The application of the method has proven successful in terms of the use of ETM+ images (in the TIR) for the identification and the analysis of the daytime UHI effect. The depiction of the source and sink landscapes, that may already be developed, records the thermal behavior of the source and sink landscapes. Monitoring of the contributions of the sink and source to the SUHI in the method proposed will have wide applicability when evaluating the SUHI at a regional scale.

Acknowledgements The support of the State Key Laboratory of Urban and Region Ecology, Research Center for EcoEnvironmental Sciences, Chinese Academy of Science, and National Natural Science Foundation of China (Grant no. 40621061) is gratefully acknowledged. Many thanks are given to all the reviewers for their constructive comments and suggestions.

\section{References}

Aniello, C., Morgan, K., Busbey, A., \& Newland, L. (1995). Mapping micro-urban heat islands using Landsat TM and a GIS. Computers and Geosciences, 21(8), 965-969.

Chander, G., \& Markham, B. (2003). Revised Landsat-5 TM radiometric calibration procedures and postcalibration dynamic ranges. IEEE Transactionson Geoscience and Remote Sensing, 41(11), 2674-2677.

Chen, L. D., Fu, B. J., \& Zhao, W. W. (2006a). Source-sink landscape and its ecological significance. Acta Ecologica Sinica, 26(5), 1444-1449.

Chen, Y. H., Wang, J., \& Li, X. B. (2002). A study on urban thermal field in Summer based on satellite remote sensing. Remote Sensing for Land and Resources, 4, 55-59.

Chen, X. L., Zhao, H. M., Li, P. X., \& Yin, Z. Y. (2006b). Remote sensing image-based analysis of the relationship between urban heat island and land use/cover changes. Remote Sensing of Environment, 104, 133-146.

Dash, P., Gottsche, F. M., Olesen, F. S., \& Fischer, H. (2002). Land surface temperature and emissivity estimation from passive sensor data: Theory and practice - Current trends. International Journal of Remote Sensing, 23(13), 2563-2594.

Dousset, B., \& Gourmelon, F. (2003). Satellite multi-sensor data analysis of urban surface temperatures and landcover. Isprs Journal of Photogrammetry and Remote Sensing, 58 (1-2), 43-54.

Gallo, K. P., \& Owen, T. W. (1998a). Assessment of urban heat island: A multi-sensor perspective for the Dallas-Ft. Worth, USA region. Geocarto International, 13, 35-41.
Gallo, K. P., \& Owen, T. W. (1998b). Satellite-based adjustments for the urban heat island temperature bias. Journal of Applied Meteorology, 38, 806-813.

Gao, B. C. (1996). NDWI - A normalized difference water index for remote sensing of vegetation liquid water from space. Remote Sensing of Environment, 58(3), 257-266.

Lo, C. P., \& Quattrochi, D. A. (2003). Land-use and landcover change, urban heat island phenomenon, and health implications: A remote sensing approach. Photogrammetric Engineering and Remote Sensing, 69(9), 10531063.

Oke, T. R. (1987). Boundary layer climates. London: Methuen.

Prata, A. J., Caselles, V., Coll, C., Sobrino, J., \& Ottle, C. (1995). Thermal remote sensing of land surface temperature from satellites: Current status and future prospects. Remote Sensing Reviews, 12, 175-224.

Purevdorj, T. S., Tateishi, R., Ishiyama, T., \& Honda, Y. (1998). Relationships between percent vegetation cover and vegetation indices. International Journal of Remote Sensing, 19(18), 3519-3535.

Roth, M., Oke, T. R., \& Emery, W. J. (1989a). Satellitederived urban heat. Remote Sensing of Environment, 86, 370-384.

Roth, M., Oke, T. R., \& Emery, W. J. (1989b). Satellite-derived urban heat islands from 3 coastal cities and the utilization of such data in urban climatology. International Journal of Remote Sensing, 10(11), 1699-1720.

Sobrino, J. A., \& Raissouni, N. (2000). Towards remote sensing methods for land cover dynamics monitoring:Application to Morocco. International Journal of Remote Sensing, 21, 353-366.

Stathopoulou, M., Cartalis, C., \& Petrakis, M. (2007). Integrating CORINE land cover data and Landsat TM for surface emissivity definition: an application for the urban area of Athens, Greece. International Journal of Remote Sensing, 28, 3291-3304.

Streutker, D. R. (2002). A remote sensing study of the urban heat island of Houston, Texas. International Journal of Remote Sensing, 23(13), 2595-2608.

Streutker, D. R. (2003). Satellite-measured growth of the urban heat island of Houston, Texas. Remote Sensing of Environment, 85, 282-289.

Voogt, J. A., \& Oke, T. R. (2003). Thermal remote sensing of urban areas. Remote Sensing of Environment, 86, 370-384.

Wang, Q. X., Wang, Z. F., \& Guo, H. (2006). the study of Urban Island in Beijing city. Climatic and Environmental Research, 11(5), 627-636.

Weng, Q. (2001). A Remote Sensing \& GIS evaluation of urban expansion and its impact on surface temperature in Zhujiang Delta, China. International Journal of Remote Sensing, 22(10), 1999-2014.

$\mathrm{Xu}$, H. Q. (2005). A Study on information extraction of water body with the modified normalized difference water index (MNDW1). Journal of Remote Sensing, 9(5), 589-595.

Zha, Y., Gao, J., \& Ni, S. (2003). Use of normalized difference built-up index in automatically mapping urban areas from TM imagery. International Journal of Remote Sensing, 24 (3), 583-594. 